\title{
In silico studies on novel inhibitors of MERS-CoV: Structure-based pharmacophore modeling, database screening and molecular docking
}

\author{
Awwad A Radwan ${ }^{1,2 *}$, Fares K Alanazi ${ }^{1}$ \\ 1Kayyali Chair, College of Pharmacy, King Saud University, PO Box 2457, Riyadh 11451, Saudi Arabia, 2Department of \\ Pharmaceutical Organic Chemistry, Faculty of Pharmacy, Assiut University, Assiut-71527, Egypt \\ *For correspondence: Email: dhna_2001@hotmail.com; Tel: +966-0505193925
}

\begin{abstract}
Purpose: To search for novel scaffolds as potential inhibitors of 3CLpro protease enzyme and as antiviral drugs.

Methods: $\mathrm{NCl}$ database was screened using structure-based pharmacophore modeling, database screening and molecular docking. Also, Lipininski's rule of 5 was applied in order to test the druglikeness of the retrieved compound. Pharmacophore modelling and subsequent post-docking analyses were used for comparison of the binding mode of the retrieved hits with that of the x-ray inhibitor, R30, against MERS-CoV 3CLpro enzyme.

Results: Five compounds were identified as potential agents for the treatment of corona virus, MERSCoV, which showed similar binding to MERS-CoV 3CLpro like that of the x-ray inhibitor, R30. As protease enzyme plays an indispensable role during virus life cycle, CoV $3 C L$ pro has been reported as a highly validated drug target and it is considered viable for the design of broad spectrum inhibitors. The selected five hit compounds bind to MERS-CoV $3 C L$ pro in a manner similar to that of the x-ray inhibitor, R30, and showed pharmacophore-fit and docking score values higher than those of R30, MERS-CoV 3CLpro-inhibitor.

Conclusion: The retrieved five hits are proposed as new scaffolds for further evaluation and optimization of their activity against MERS-CoV.
\end{abstract}

Keywords: MERS-CoV pharmacophore, Molecular docking, Protease enzyme, X-ray inhibitor

\begin{abstract}
This is an Open Access article that uses a funding model which does not charge readers or their institutions for access and distributed under the terms of the Creative Commons Attribution License (http://creativecommons.org/licenses/by/4.0) and the Budapest Open Access Initiative (http://www.budapestopenaccessinitiative.org/read), which permit unrestricted use, distribution, and reproduction in any medium, provided the original work is properly credited.
\end{abstract}

Tropical Journal of Pharmaceutical Research is indexed by Science Citation Index (SciSearch), Scopus, International Pharmaceutical Abstract, Chemical Abstracts, Embase, Index Copernicus, EBSCO, African Index Medicus, JournalSeek, Journal Citation Reports/Science Edition, Directory of Open Access Journals (DOAJ), African Journal Online, Bioline International, Open-J-Gate and Pharmacy Abstracts

\section{INTRODUCTION}

In June 2012, the Middle East respiratory syndrome coronavirus (MERS-CoV) was first diagnosed from a Saudi Arabian patient who had died from progressive respiratory and renal failure [1]. Up to July 2015, it had infected at least 1401 people with a fatality rate surpassing $39 \%$
[2]. Recent reports have confirmed the humanto-human transmission of MERS-CoV. Thus, prompt efforts are necessary to develop new antiviral drugs or therapy for treatment of MERSCoV.

Protease enzyme is essential for viral replication by mediating the maturation of viral replicase 
polyprotein by the viral papain-like protease (PLpro) and the 3-chymotrypsin-like protease (3CLpro) thus, these proteases become alternative targets for potential antiviral drugs. Since the 3CLpro and PLpro enzymes are unique in the virus, present only in the virus and not in the host cell, this therapeutic strategy appears to be much safer than the one that targets host cell proteins [3,4]. Viral proteases, including the papain-like protease (PLpro) and the main protease 3C-like protease (3CLpro), process the replication of the genomic RNA of MERS coronavirus.

Since 2012, there are no effective antiviral therapeutic against human coronaviruses, including MERS-CoV but only primary organ support for respiratory and renal failure were the only clinical management of the infection. Use of broad-spectrum antivirals including antiviral agents against influenza, has also been recommended [5]. Treatment with interferon has also been suggested as a promising therapeutic strategy against MERS-CoV [6]. In 2013, first generation of protease inhibitor antiviral drugs, such as N3 [3] and CE-10 [7] that were optimized for inhibition of SARS-CoV 3CLpro have been reported to block the activity of MERS-CoV 3CLpro. Some SARS-CoV 3CLpro peptidomimetics which contains a Michael acceptor, (i.e., $\alpha, \beta$-unsaturated carbonyl) [1], or contains activated carbonyl functionality displayed inhibition against MERS-CoV 3CLpro.

In view of these findings there is still a need for novel potent MERS-CoV inhibitors. Traditional synthesis and screening of a series of new compounds is well known as cost and time consuming process. On the other hand, an alternative process can be used by applying in silico screening of small molecule databases for novel compounds. Dependent on the amount of information available, either ligand-based or structure-based molecular design can be applied to find lead molecules [8]. In this work we describe screening a set of compounds from National Cancer Institute (NCI) database against MERS-CoV 3CLpro protein, with bound ligand R30 [9], by utilizing a ligandScout program [10] and AutoDock Vina program [11].

\section{METHODS}

\section{Preparation of the protein structure and ligand structures}

The National Cancer Institute $(\mathrm{NCl})$ database, a free database for virtual screening, contains about 250,250 compounds in 3D formats. The $x-$ ray crystal structure of MERS-CoV 3CLpro bound by the ligand, $\mathrm{N}-\{4-[(1 \mathrm{H}-$ benzotriazol-1ylacetyl)(thiophen-3-ylmethyl)amino]phenyl\}propanemide (R30), was obtained from the Protein Data Bank (PDB code: 4YLU) [9]. The undertaken molecular modelling works were done on PC Windows 7 Home Premium Intel(R) Core(TM)2 Duo, 1.83GHz.

\section{Structure-based pharmacophore building}

The spatial arrangement of atoms or functional groups, called a pharmacophore, suggests how a ligand molecule can interact inside the binding site of a target protein.

The pharmacophore theory is based on the fact that ligand molecules that have the same biological binding site, should have common pharmacophore [12]. Based on the available information of ligands and the target macromolecule, there are two pharmacophore modelling approaches. The ligand-based approach is carried out by extracting common spatial arrangement of the chemical features of a set of known ligands postulated to have same binding mode to a specific target protein. While, the structure based approach demands necessitates the 3D structure of a target molecule or a macromolecule-ligand complex.

Using LigandScout program [10], the latter approach was utilized to build a pharmacophore model making use of the x-ray crystal structure of MERS-CoV 3CLpro bound with inhibitor compound R30 (PDB code: 4YLU) [9]. In this study, the pharmacophore model is obtained through the investigation of the complementary chemical features of the binding site and their 3D-arrangement around the bound ligand.

\section{Docking protocol}

The coordinates of the $\mathrm{x}$-ray structure of the MERS-CoV 3CLpro (4YLU) bound to its ligand (R30) were got from the Protein Data Bank (PDB) [9]. The x-ray ligand was redocked into its binding pocket to examine whether the docked pose could have same orientation as the x-ray ligand indicating the validity of the parameters in the docking simulation and in reproducing the $x-$ ray crystal structure. The AutoDock Vina program was used for molecular docking-based virtual screening, VSDK.

\section{Virtual screening}

The coordinate files of ligands datasets in the $\mathrm{NCl}$ database are filtered by Lipinski's rule. Only the molecules which in accordance with Lipinski's rule can be considered as hits, retrieved from $\mathrm{NCl}$ database and grouped in a 
chemical library. The obtained library was subjected to virtual screening using structurebased pharmacophore model resulting in a reduced size chemical library. The candidates in the obtained library were ranked based on docking score using AutoDock Vina program. Top ranked candidates were suggested for experimental screening as potential therapeutic agents for treatment of MERS-CoV.

\section{RESULTS}

Lipinski's rule of 5 was used for filtering the $\mathrm{NCl}$ database (250,250 entries) and identified a reduced diversity subset including 3120 small molecules that were subjected to further screening, using macromolecule-ligand-complexbased pharmacophore model (Figure 1 and Figure 2) and using docking study (Table 1, Figure 3).

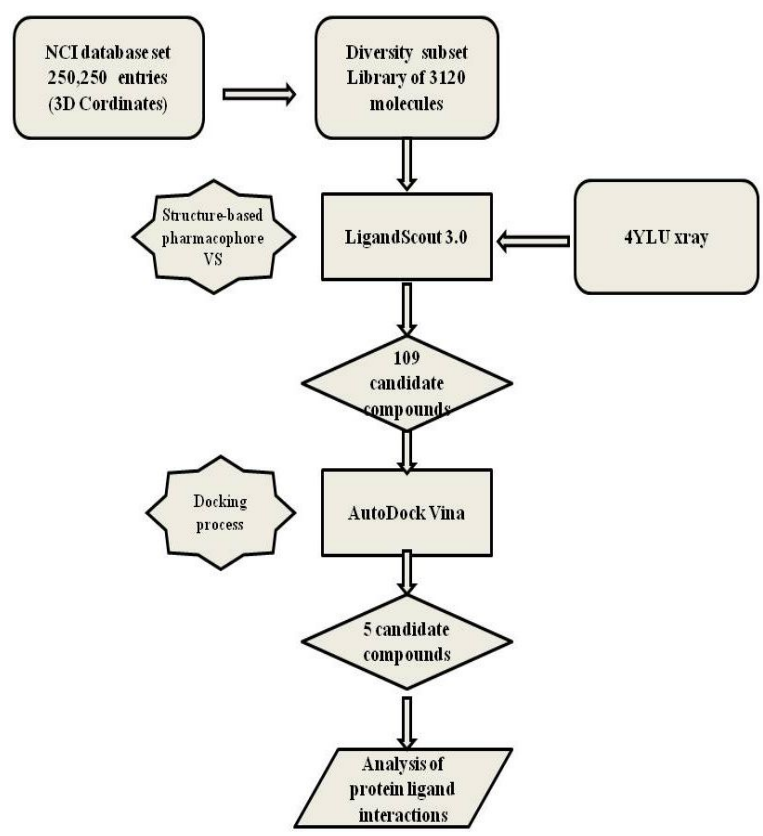

Figure 1: Schematic representation of the structurebased virtual screening work-flow

Docking study was undertaken in order to screen the hits retrieved from virtual screening using the pharmacophore query. The target macromolecule file (PDB ID: 4YLU) was obtained from PDB (www.rcsb.org) and explored the binding mode of peptide-like-inhibitor, $\mathrm{N}-\{4-[(1 \mathrm{H}-$ benzotriazol-1-ylacetyl)(thiophen-3-

ylmethyl)amino]phenyl\}propanamide, R30, in the binding pocket (Figure 4).

\section{DISCUSSION}

Figure 1 shows the schematic representation of screening workflow. Lipinski's Rule of Five describes the molecular characteristics crucial for in vivo drug's pharmacokinetics, involving their absorption, distribution, metabolism, and elimination (ADME). The rule determines whether a biologically-active molecule would be a likely orally active drug or not. First, Lipinski's rule of 5 was used for filtering the $\mathrm{NCl}$ database (250,250 entries) and identified a reduced diversity subset including 3120 small molecule. We screened these 3120 compounds using macromolecule-ligand-complex-based pharmacophore modelling. Using the 3D structure of the MERS-CoV 3CLpro-ligand complex (4YLU.pdb), LigandScout program analyse the spatial arrangement of the matching chemical characteristics of the binding site and design a pharmacophore model with the selected features. The resulting pharmacophore model is composed of four features including two hydrophobes, one hydrogen acceptor, and one hydrogen donor, features, as shown in Figure 2.

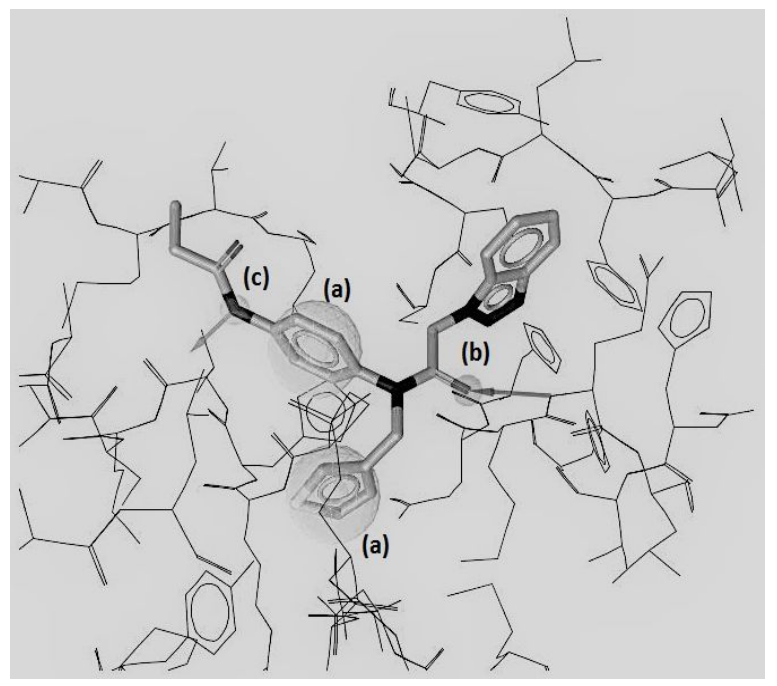

Figure 2: The macromolecule-ligand-complex-based pharmacophore model shows two hydrophobes (a), one hydrogen acceptor (b) and one hydrogen donor (c)

Table 1: Binding energy (Kcal/mol) and hydrogen bonds of the selected hit compounds

\begin{tabular}{|c|c|c|c|}
\hline Compound & $\begin{array}{c}\text { Binding } \\
\text { energy } \\
\left(\mathrm{kcal}^{-1}\right. \\
\left.\text { mol }^{-1}\right) \\
\end{array}$ & $\begin{array}{l}\text { No. of H- } \\
\text { bonds }\end{array}$ & $\begin{array}{l}\text { Interacting } \\
\text { residues }\end{array}$ \\
\hline NSC648199 & -8.7 & 2 & $\begin{array}{l}\text { Gln167, } \\
\text { Phe143 }\end{array}$ \\
\hline NSC159375 & -8.3 & 1 & Glu169 \\
\hline NSC29007 & -8.1 & 1 & Gly146 \\
\hline NSC335985 & -8.1 & 2 & $\begin{array}{l}\text { Ser147, } \\
\text { Glu169 }\end{array}$ \\
\hline NSC337571 & -7.8 & 0 & \\
\hline $\begin{array}{l}\text { R30 (x-ray } \\
\text { ligand) }\end{array}$ & -7.6 & 3 & $\begin{array}{l}\text { Tyr54, } \\
\text { His166, } \\
\text { Glu169 }\end{array}$ \\
\hline
\end{tabular}




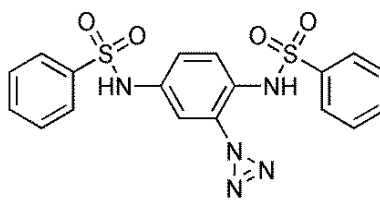

NSC29007

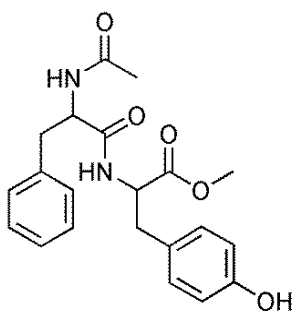

NSC337571

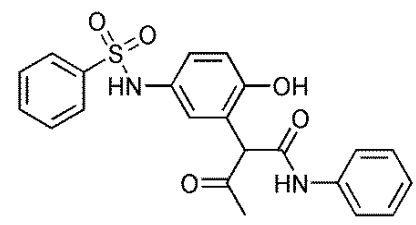

NSC159375

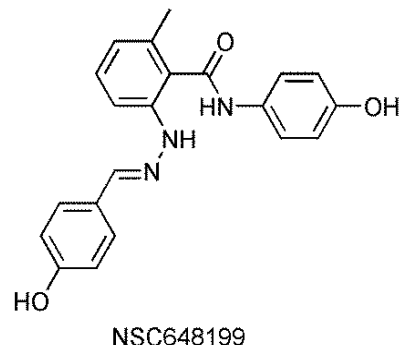

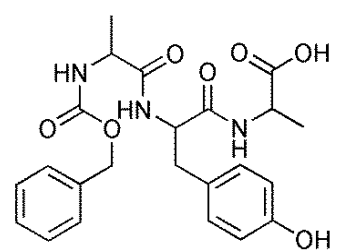

NSC335985

Figure 3: 2D chemical structures of the selected hit compounds

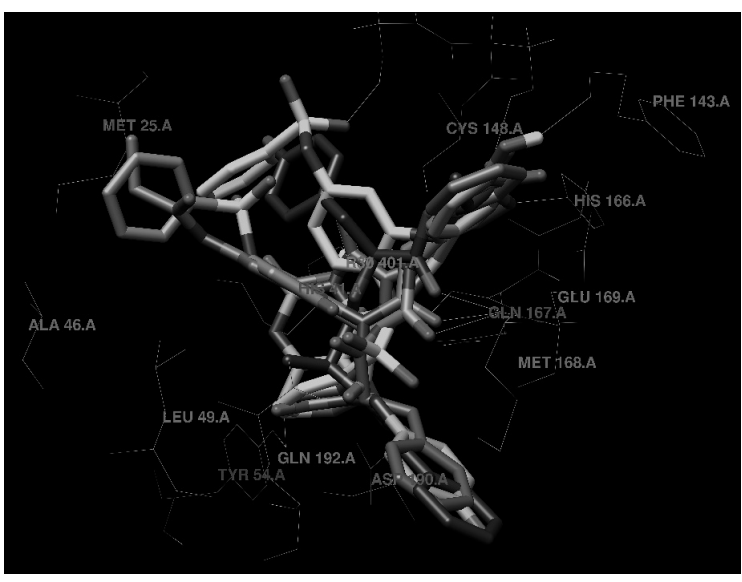

Figure 4: The 3D diagrams of interaction between the MERS-CoV 3CLpro and the five hit compounds overlaid on R30 x-ray ligand (colored black). The dotted lines show the hydrogen bond between the compounds and the binding.

Based on the pharmacophore fit value, the top 109 ligands were suggested for further screening using AutoDock Vina program. The AutoDock Vina algorithm searches different orientations of the compounds inside the binding site of receptor. Rely on the binding energy scores, five ligands were selected, NSC159375, NSC29007, NSC337571, NSC335985, NSC648199 (Table 1, Figure 3 ) were having lower energy scores than that R30 (xray ligand) which reveal promising binding affinity towards the active site of MERS$\mathrm{CoV}$ 3CLpro. These ligands might act as potential inhibitors for the MERS-CoV 3CLpro and were suggested for experimental screening.

Docking study was undertaken using AutoDock Vina in order to screen the hits retrieved from virtual screening using the structure-based pharmacophore model. Also, the possible interactions between the top-score ranked hits and the active site of the MERS-CoV 3CLpro were compared with the binding mode of the known 4YLU peptide-like-inhibitor, $\quad \mathrm{N}-\{4-[(1 \mathrm{H}-$ benzotriazol-1-ylacetyl)(thiophen-3-

ylmethyl)amino]phenyl\}propanamide, R30. R30 has a tricyclic structure and is oriented in its binding site that is lined with the side chains of Met25, His41, Ala46, Leu49, Tyr54, Phe143, Cys148, His166, Met168, Glu169, Asp190, Gln192 (Figure 4, Table 1).

One of the nitrogen atoms of the benzotriazole moiety form hydrogen bond to the imidazolenitrogen of His166, while the carbonyl oxygen of the acetyl moiety from a hydrogen bond to the amide-nitrogen of Glu169. Replication of the binding mode of the bound inhibitor, R30, by the docking algorithm used in the virtual screen would indicate that it is well calibrated for this class of receptor.

\section{CONCLUSION}

A computational approach using pharmacophore modelling and docking study has successfully applied to screen $\mathrm{NCl}$ database against MERSCoV 3CLpro structure in order to discover new inhibitors of MERS-CoV. Using the structurebased screening approach, five compounds are identified as potential agents for the treatment of corona virus, MERS-CoV. Binding mode analyses reveal that the way these compounds bind to MERS-CoV 3CLpro is the same as that of the $x$-ray inhibitor, R30. The results suggest that these molecules can possibly be developed as novel lead compounds in anti-MERS-CoV drug design. 


\section{DECLARATIONS}

\section{Acknowledgement}

The authors express sincere appreciation to the deanship of Scientific Research at King Saud University for its funding of this research through the research Group Project no. RGP-1435-080. The author (F. K. A.) would like to thank the Kayyali chair for their support.

\section{Conflict of interest}

No conflict of interest is associated with this work.

\section{Contribution of authors}

This work was done by the author(s) named in this article and all responsibilities relating to the content of this article will be borne by the authors. Awwad A Radwan conceived and designed the study, Awwad A Radwan and Fares $\mathrm{K}$ Alanazi gathered and evaluated the data, Awwad A Radwan and Fares K Alanazi wrote read and approved the manuscript for publication.

\section{REFERENCES}

1. Zaki AM, van Boheemen S, Bestebroer TM, Osterhaus ADME, Fouchier RAM. Isolation of a novel coronavirus from a man with pneumonia in Saudi Arabia. N Engl J Med 2012; 367: 1814-1820.

2. Pillaiyar T, Manickam M, Jung S-H. Middle East Respiratory Syndrome-Coronavirus (MERS-CoV): An Updated Overview and Pharmacotherapeutics. Med Chem 2015; 5: 361-372.

3. Ren Z, Yan L, Zhang N, Guo Y, Yang C, Lou Z, Rao Z. The newly emerged SARS-like coronavirus HCoV-EMC also has an 'Achilles' heel': current effective inhibitor targeting a 3C-like protease. Protein Cell 2013; 4: 248250.
4. Yang $H$, Xie $W$, Xue $X$, Yang $K$, Ma J, Liang $W$, Zhao Qi, Zhou Z, Pei D, Ziebuhr J, Hilgenfeld R, Yuen KY, Wong L, Gao G, Chen S, Chen Z, Ma D, Bartlam M. Design of wide-spectrum inhibitors targeting coronavirus main proteases. PLoS Biol 2005; 3: e324.

5. Chan JF, Li KS, To KK, Cheng VC, Chen H, Yuen KY. Is the discovery of the novel human betacoronavirus 2C EMC/2012 (HCoV-EMC) the beginning of another SARS-like pandemic? J Infect 2012; 65: 477-489.

6. De Wilde AH, Raj VS, Oudshoorn D, Bestebroer TM, van Nieuwkoop S, Limpens RW, Posthuma CC, van der Meer Y, Bárcena M, Haagmans BB, Snijder EJ, van den $B G$, Hoogen BG. MERS-coronavirus replication induces severe in vitro cytopathology and is strongly inhibited by cyclosporin $A$ or interferon- $\alpha$ treatment. J Gen Virol 2013; 94: 1749-1760.

7. Kilianski A, Mielech A, Deng X, Baker SC. Assessing Activity and Inhibition of MERS-CoV Papain-like and $3 C$ like Proteases Using Luciferase-based Biosensors. J Virol 2013; 87: 11955-1962.

8. Lengauer $T$, Lemmen $C$, Rarey M, Zimmermann $M$. Novel technologies for virtual screening. Drug Discov Today 2004; 9: 27-34.

9. Tomar S, Melanie LJ, Sarah EStJ, Heather LO, Prasanth RN, Lake NP, Arun KG, Mark RD, Andrew DM. Ligandinduced Dimerization of Middle East Respiratory Syndrome (MERS) Coronavirus nsp5 Protease (3CLpro): Implications for NSP5 regulation and the development of antivirals. J Biol Chem 2015; 290: 19403-19422.

10. Wolber G, Langer T. LigandScout: 3-D pharmacophores derived from protein-bound ligands and their use as virtual screening filters. J Chem Inf Model 2005; 45: 160-9.

11. Trott O, Olson AJ. AutoDock Vina: improving the speed and accuracy of docking with a new scoring function, efficient optimization and multithreading. J Comput Chem 2010; 31: 455-461.

12. Chen JH, Liu TL, Yang LJ, Li LL, Wei YQ, Yang SY. Pharmacophore modeling and virtual screening studies of checkpoint kinase 1 inhibitors. Chem Pharm Bull 2009; 57: 704-9. 